\title{
System for Assessing Eye Injury Potential of Propelled Objects
}

\author{
Robert E. Berger \\ Center for Consumer Product Technology, National Bureau of Standards, Washington, DC 20234 \\ September 27, 1978

\begin{abstract}
A test system is proposed to evaluate the ocular injury potential of propelled objects. The object in question is fired into a thin rubber pad, and the force of impact and the rise time are measured. For a set of test projectiles, the response of the system was shown to correlate with the likelihood of injury, as predicted by a mathematical model. The response was further related to ocular injury tolerance curves which were generated by the math model using data from impact injuries to real eyes.
\end{abstract}

Key words: Eye injury; impact; ocular contusion; projectiles; propelled objects; test methods; toy safety.

\section{Introduction}

Injuries from consumer products result from a combination of factors, including the design and manufacture of the product and the way people interact with it. The examination of injury data provides clues for the relative involvement of these factors, and the clues, in turn, generally suggest methods of testing. (Standards and test methods are, of course, aimed at increased safety through reduction of the frequency and severity of product-associated injuries.) However, the relationship of test method to the real life injury situation is rarely demonstrated in an adequately logical fashion.

The primary difficulty is that, for ethical and practical reasons, the injury situation cannot be reproduced in the laboratory. The test method must, therefore, follow from well-designed simulations of the injury environment. This requires a rational approach to modelling the real life injury situation and a subsequent correlation of model (whether physical or mathematical) to laboratory test method. Since the simulation is an abstraction, it may be too complicated or non-reproducible for use in a test system for the products suspected to cause injury. Therefore, test systems for product safety must be supported by the following:

(1) a rationale for the simulation by which the injury likelihood is assessed, and

(2) a demonstrated correlation between the simulation and the final test method system.

This paper illustrates how these criteria guided the development of a system for assessing the ocular contusion injury potential of propelled objects. The need for such a system was suggested in connection with a particular class of products, toy projectiles, but the ensuing discussion is thought to be valid for all low speed (much less than ballistic velocities) propelled objects.
A variety of ocular contusion injuries resulting from blows to the eye have been reported; these include chamber angle irregularities such as ruptures of the iris root (iridodialysis) $[1]^{1}$, trabecular meshwork damage (leading to gradual development of glaucoma) [2], retinal dialyses [3], and displacements of the lens [4] (see fig. 1). All of these effects of trauma have been related to the mechanism of eyeball expansion perpendicular to the direction of impact [3], [4].

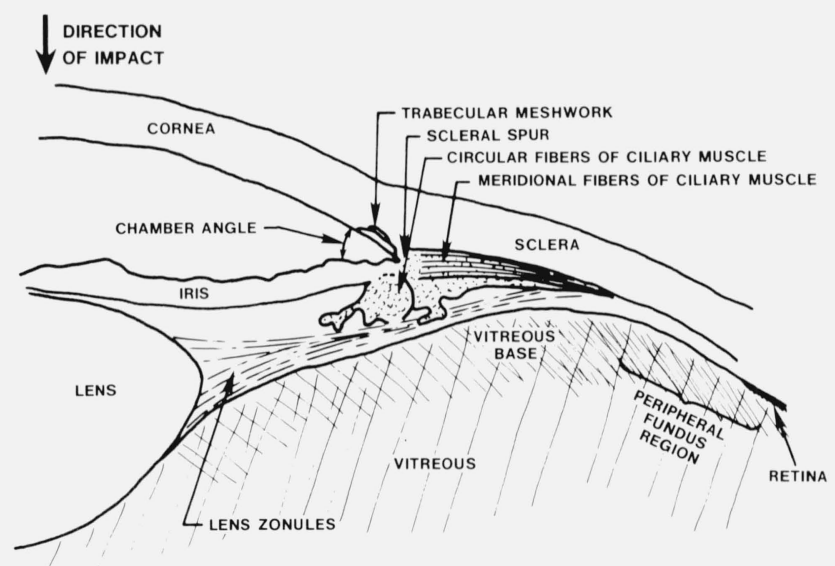

FIGURE 1. Sketch of areas of eyeball which sustain injury during impact.

The rate of loading has also been found to be an important factor: the likelihood of injury increases with rate of loading [3].

In an earlier study, a mathematical model was developed to predict the force of impact between a missile and the eye [5]. The model was based on the theory of quasi-static,

\footnotetext{
${ }^{1}$ Figures in brackets indicate the literature references at the end of this paper.
} 
elastic impact, which was justified by the relatively slow speeds of typical toy projectiles. The maximum force, $f$, between a projectile and the eye was chosen as an injury parameter because it was experimentally shown to be related to the expansion of the eyeball normal to the impact direction. $f$ is calculated as follows [5]:

$$
f=\frac{1.69 T_{0}^{3 / 5} D^{* 1 / 5}}{\left(k_{1}+k_{2}\right)^{2 / 5}}
$$

where $T_{0}=1 / 2 M V^{2}$ is the initial kinetic energy of the projectile with mass $\mathrm{M}$ and velocity $V . D^{*}$ is a geometric parameter defined by

$$
D *=\frac{d D}{d+D}
$$

where $d$ is the effective diameter of the projectile tip and $D$ is the diameter of the eye. $k_{1}$ and $k_{2}$ are material parameters of the projectile and eye respectively,

$$
k_{i}=\frac{1-\nu_{i}{ }^{2}}{E_{i}},
$$

where $E_{i}$ and $\nu_{i}$ are the elastic modulus and Poisson's ratio, respectively; $i=1$ for projectile, $i=2$ for eye. Based on previous studies reported in the literature, the following material and geometric constants for the eye appear to be reasonable [5]:

$$
\begin{aligned}
E_{2} & =3.75 \times 10^{7} \text { dynes } / \mathrm{cm}^{2} \\
\nu_{2} & =0.5 \\
D & =2.5 \mathrm{~cm}
\end{aligned}
$$

The elapsed time, $t$, between the onset of impact and the time of maximum force represents the rate of load and can be computed by [5]:

$$
t=2.47 \mathrm{Y} / \mathrm{V}
$$

where $Y$, the maximum relative displacement, is given by

$$
Y=\frac{1.04 f^{2 / 3}\left(k_{1}+k_{2}\right)^{2 / 3}}{D^{* 1 / 3}}
$$

If the projectile characteristics, $M, V, d$ and $k_{1}$ are specified, the force, $f$, and the time to maximum load, $t$, can be calculated. It may be noted that $k_{1} \rightarrow 0$ for rigid projectiles.

This model is then considered to be the simulation with which the likelihood of contusion injury is assessed.

Tolerance curves for eye injury were determined by applying the mathematical model to projectile impacts which caused injury to real eyes [5], as reported in the ophthalmologic literature. These curves are shown in figure $2 . F$ is the non-dimensional force

$$
F=\frac{f}{E_{2} D^{2}}
$$

which was used instead of $f$ to compare eye injuries from different species. The safety factor 2 curve is relative to chamber angle damage which is considered to represent the threshold for clinically detectable contusion injury. These tolerance curves, as they relate to a set of projectile toys, were also shown to agree with the subjective opinions of a panel of ophthalmologists [5].

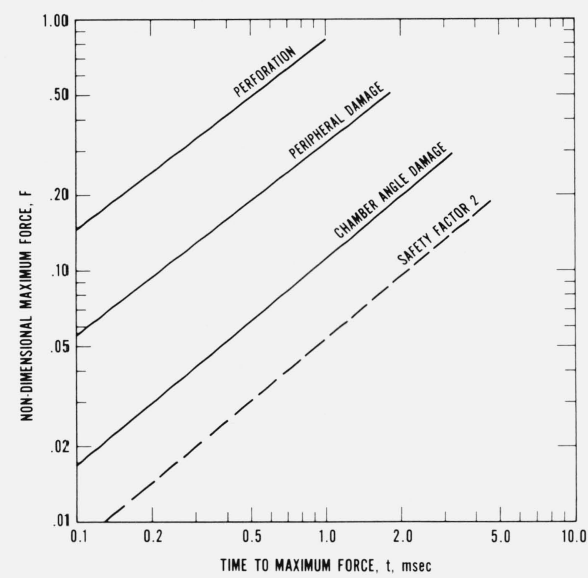

Figure 2. Ocular contusion tolerance curves, reference 5 .

\section{Materials and Methods}

It was suggested earlier [5] that a simple impact test system, where projectiles are fired into a test pad and where the force of impact is recorded with appropriate transducers, may provide a suitable correlation with the predictions of the model. To evaluate this correlation, a set of well defined projectiles was fired into each of a set of candidate test systems, and the response of the system was measured, as well as the important characteristics, $M, V, d$, of the projectiles. The model was then used to calculate $F$ and $t$, which was compared to the experimental results (see fig. 3).

\subsection{Projectiles}

A system was designed so that projectiles could be accurately impacted against the target of any test system. Test projectiles were composed of several components (fig. 4):

1. A hollow cylindrical nylon guide (diameter $0.635 \mathrm{~cm}$, length $4.05 \mathrm{~cm}$ ) of mass $0.89 \mathrm{~g}$ traveled in a linear ball 


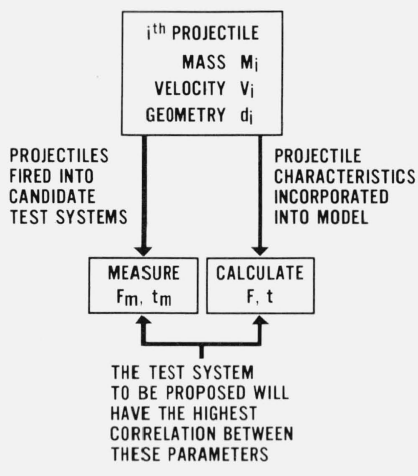

Figure 3. Schematic for determining a suitable test system.

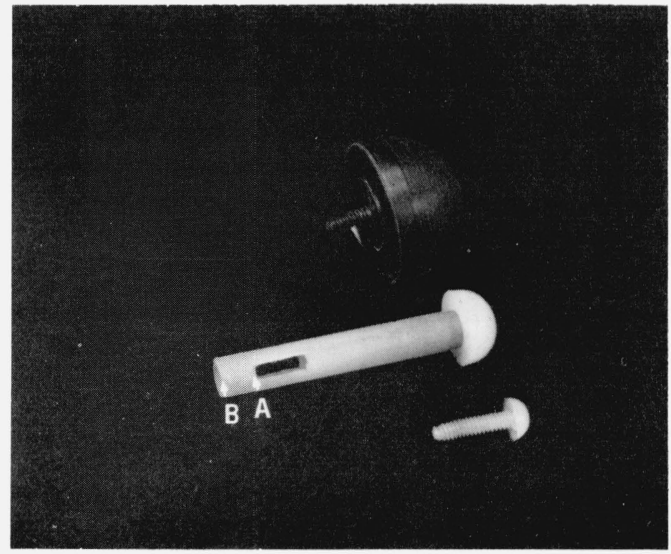

(a)

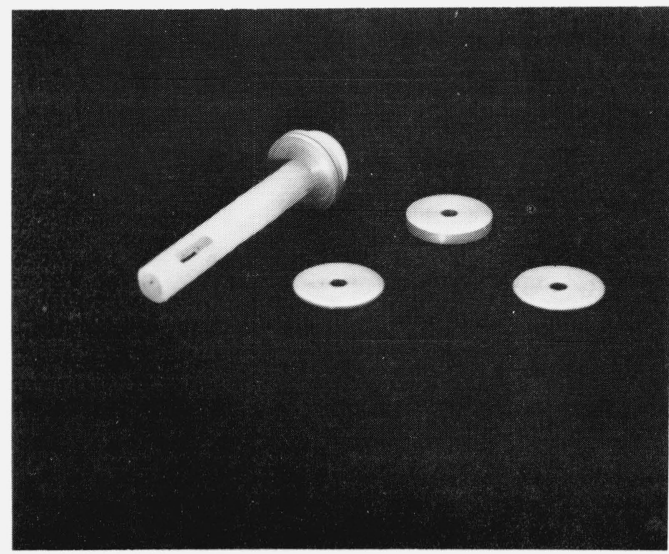

(b)

FigurE 4. Test projectiles.

(a) nylon guide with hemispherical caps; distance $A B$ used in velocity measurements. (b) weights used to increase mass of projectile. bushing on the missile launcher (described below) to maintain accurate impacts. The leading edge of the tube, which was not bored through, was drilled and tapped to accommodate a set of hemispherical caps. A window was cut into the tube near the trailing edge for use in velocity measurements.

2. A set of nylon hemispherical caps of radii 0.317 , 0.635 , and $1.27 \mathrm{~cm}$, with masses $0.13,0.87$, and $7.07 \mathrm{~g}$, respectively, could be screwed into the guide by means of threaded studs which were an integral part of the caps.

3. A set of 5 brass weights with masses $0.67,1.09,1.80$, 2.04 , and $3.25 \mathrm{~g}$ could be inserted on the screw thread between the guide tube and the caps. The total mass of the projectile could then be varied by using the brass weights in different combinations.

The missile launcher is shown in figure 5. A plunger rides in an aluminum support tube and is attached to a spring which may be compressed to three alternate stop positions. When released from the stop, the spring uncoils and causes the plunger to impact the nylon guide end of the projectile. The nylon guide then advances in a linear ball bushing of length $1.905 \mathrm{~cm}$ which was mounted within the aluminum support tube and stays in the bearing during its impact with the test system. The distance traveled by the projectile was about $2-3 \mathrm{~cm}$. Within this displacement range, the response of the test system (described later) was virtually independent of travel distance.

The velocity of the projectile is measured by a meter which records the time interval required for edges $\mathrm{A}$ and $\mathrm{B}$, of the guide tube (fig. 4), to interrupt a light beam. A window in the aluminum support tube allows the beam to penetrate the window in the projectile guide tube. The velocities achieved by the projectiles depend on the stop position and the mass of the projectile. Velocities can be further varied by adjusting the tension on the spring with the screw at the rear of the aluminum support tube. The lightest projectile, about $1 \mathrm{~g}$, achieved velocities up to $25 \mathrm{~m} / \mathrm{sec}$; the heaviest projectiles, about $10 \mathrm{~g}$, achieved velocities up to $7 \mathrm{~m} / \mathrm{sec}$.

It was felt that the range of parameters observed on toys [5], [6] would be well represented by varying tip geometry, mass, and velocity through the use of various combinations of the above described parameters.

\subsection{Impact Assessment Systems}

Four disc-shaped impact pads with accompanying support structures were constructed. Three were natural rubber gum compounds (ASTM 3184-73, compound 1A), Shore A hardness of 36 , in thicknesses of $1.02,1.91$, and $3.81 \mathrm{~cm}$. The fourth was a rubber carbon black compound (ASTM D15-73, compound 7A), Shore A hardness, 50, $1.02 \mathrm{~cm}$ thick. The diameter of each pad was $5.08 \mathrm{~cm}$, which was found to be sufficiently large that end effects were negligible. 


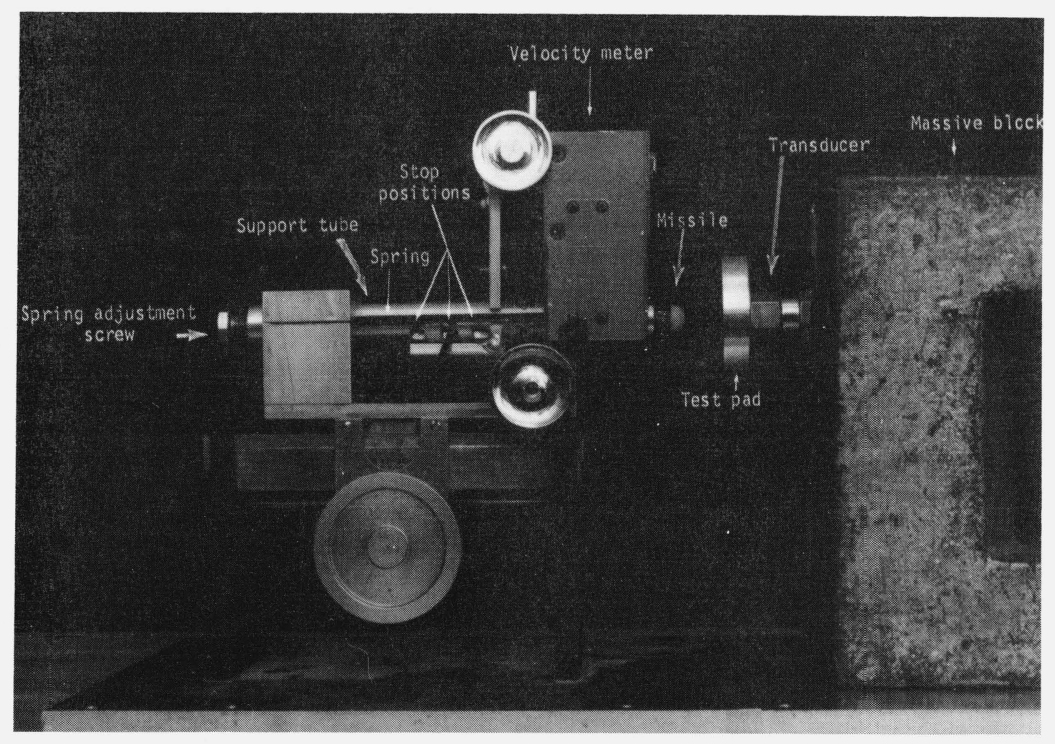

Figure 5. Missile launching system.

A typical support structure is shown in figure 6. These supports were made of aluminum and the thickness was the same as the thickness of the particular pad for which it was

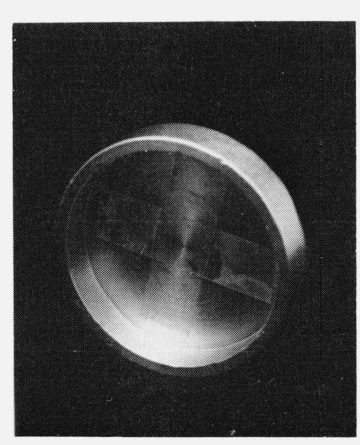

(a)

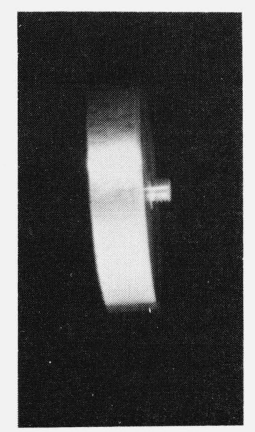

(b)

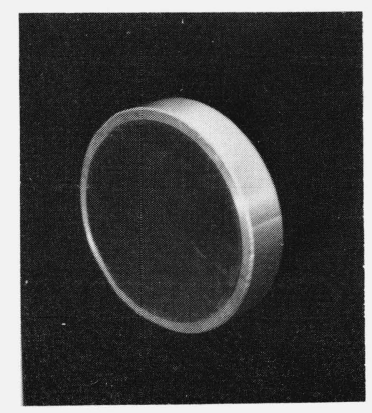

(c)

FIGURE 6. Support structure for test pads, double stick tape used to maintain contact between pad and support. made. The plateau and screw thread at the back is designed for attachment to a force transducer. The purpose of the support is to hold the pad during impact by projectiles. It must be sufficiently rigid so as not to deform during impact and so that it will transfer the force of impact to the transducer.

In preliminary experiments, undesirable vibrations were observed which were traced to the presence of air gaps between the pad and the surface of the support structure. It is therefore necessary to ensure that the pad and its support are in intimate contact. The use of double stick tape between the two surfaces was found to provide a satisfactory solution.

The central part of the impact test system is then considered to be a pad with its accompanying support structure. Any instrumentation which is capable of reliably recording the force history on the support structure is appropriate for use with the test system. For this study, a piezo-electric force transducer (Endevco, model No. 2103) was connected to the aluminum support structure. The transducer was, in turn, rigidly attached to the massive block $(22.7 \mathrm{~kg})$ shown in figure 4 . This effectively infinite mass is required to ensure that all of the impact energy is used in deforming the pad rather than accelerating the support structure. The signal from the force transducer was first sent to a signal conditioner and amplifier (Endevco, model No. 4470/4472.1), and then to a storage oscilloscope where the force-time history of any impact could be displayed.

For every combination of projectile mass, $M$, and tip diameter, $d$, which impacted the set of test pads the velocity, $V$, was measured. Then, using eqs. (1) to (4), $F$ and $t$ were calculated using the assumption that the missiles are rigid compared to the eye. For the same impact, the maximum 
force recorded by the force transducer, $F_{m}$, and the elapsed time to maximum force, $t_{m}$, were also read from the oscilloscope trace.

\section{Results and Discussion}

\subsection{Preliminary Experiments}

In order to assess the performance of the test pads and the relation of measured forces and time to the quasi-static theory, a few impact experiments were conducted by dropping steel balls onto the test system from various test heights.
In this way, extraneous effects which might be introduced by the missile launching system could be eliminated. Oscilloscope traces from these experiments are shown in figures 7 and 8 for a $1.27 \mathrm{~cm}$ diameter ball and a $0.635 \mathrm{~cm}$ diameter ball, respectively.

Each trace may be described as the superposition of two distinct modes: wave propagation (vibration) and static-like contact. The relative importance of each depends upon how nearly the impact approaches the quasi-static situation. When the duration of contact is long compared to the transit time for wave motion, the energy used in wave propagation is small compared to the initial kinetic energy [7]. Then the
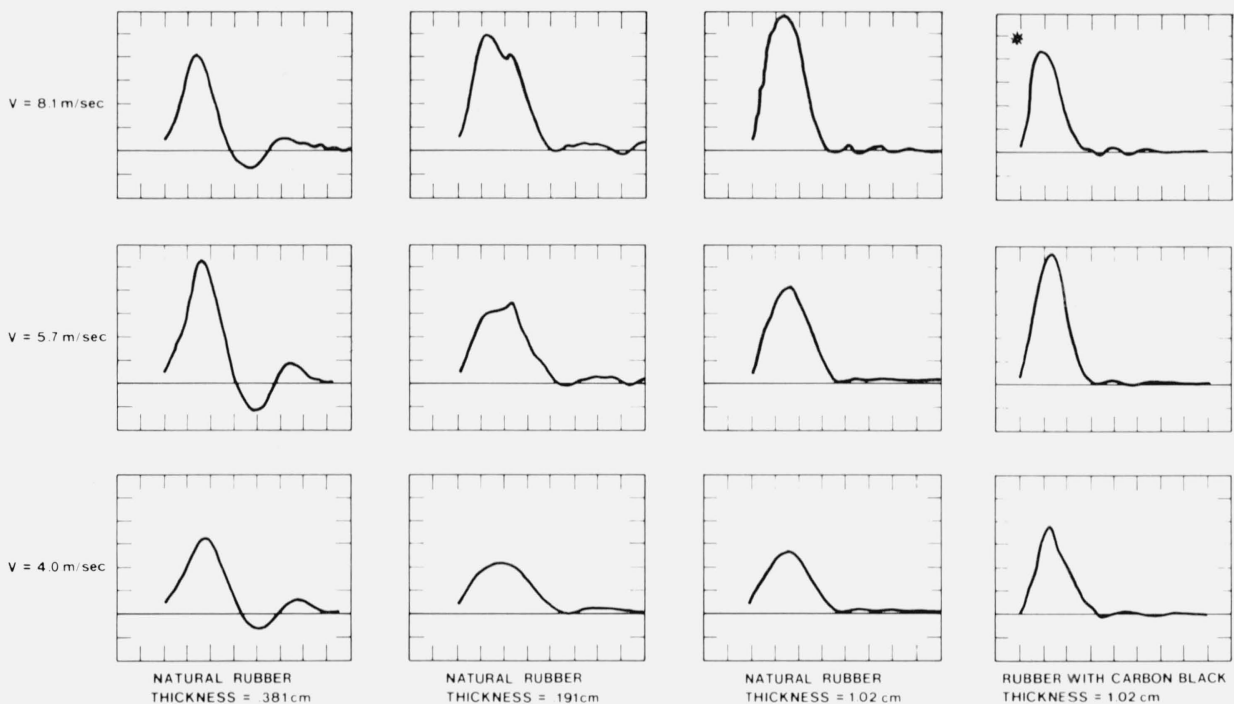

FIGURE 7. Sketches of oscilloscope traces from ball drop experiments with various test pads, ball diameter $=1.27 \mathrm{~cm}$. All vertical scales are $26.7 \mathrm{~N}$ per division, except where marked with an asterisk (then $53.4 \mathrm{~N} /$ div). All horizontal scales are $0.5 \mathrm{msec}$ per division.
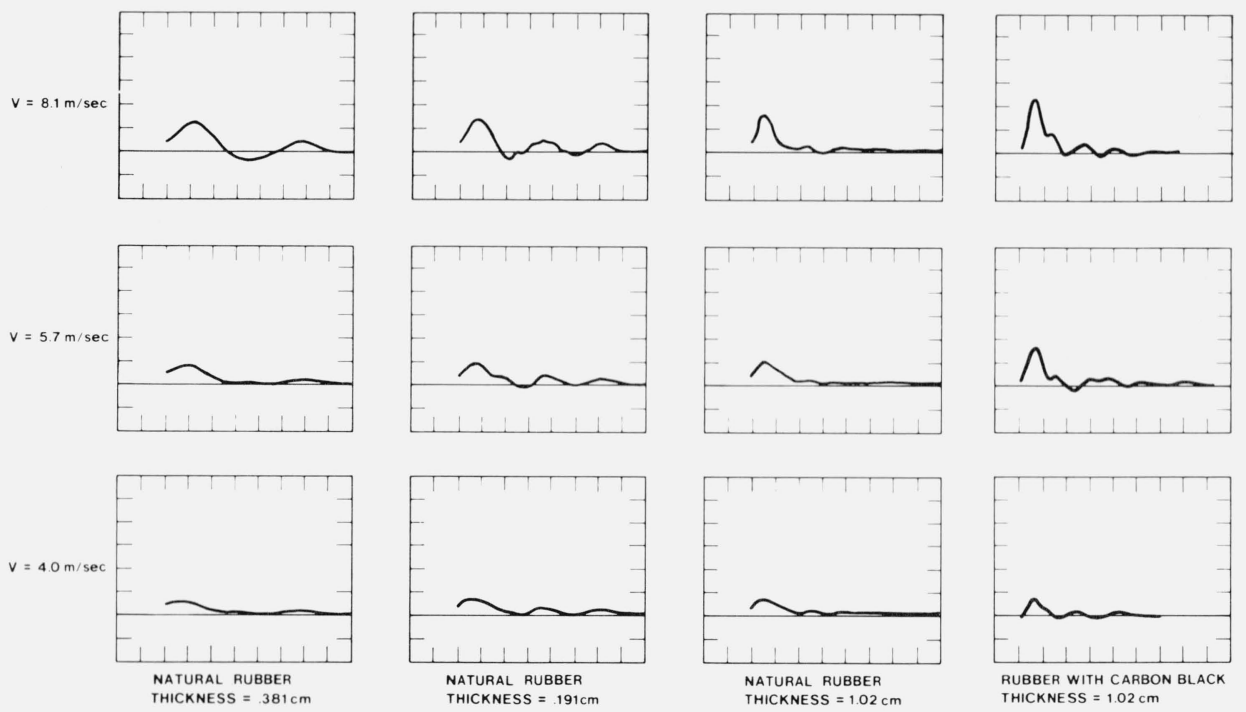

FIGURE 8. Sketches of oscilloscope traces from ball drop experiments with various test pads, ball diameter $=.635 \mathrm{~cm}$. All vertical scales are $27.6 \mathrm{~N}$ per division; all horizontal scales are $0.5 \mathrm{msec}$ per division. 
quasi-static theory is considered to be valid, and the resulting deformations are the same as if the configuration were instantaneously static. For the traces of figures 7 and 8, the initial pulse may be considered to be related to the staticlike deformation, while those which follow it are typical of the vibrational wave motion. As the duration of these vibrational pulses increases compared to the initial pulse, more energy is associated with vibration.

The time between the peaks of the vibrational pulses may be approximated by $2 T / C_{o}$, where $T$ is the pad thickness and $C_{o}$ is the velocity of elastic wave travel given by

$$
C_{o}=\left(\frac{E}{\rho}\right)^{1 / 2}
$$

where $E$ and $\rho$ are the elastic modulus and density of the rubber, respectively. Therefore, the thinner and harder the pad, the smaller the transit time for wave propagation and the more valid the quasi-static theory. This is desirable since the model to predict ocular injury is based on the quasi-static theory; the experimental results of figures 7 and 8 agree with these predictions. For the natural rubber, $3.81 \mathrm{~cm}$ thick pad, the vibrational pulse durations are of the same order of magnitude as the initial pulse. Consequently, a large amount of energy is used in wave propagation; in fact, this mode may dominate. For the $1.91 \mathrm{~cm}$ pad, there is apparently some interaction between the two modes, causing the duration of the initial pulse to be extended. The other two pads are so thin that the wave propagation effects appear to be small.

The duration of contact can also be calculated from the quasi-static theory. From eqs. (1) to (3) it is seen that

$$
t \propto \frac{M^{2 / 5}}{V^{1 / 5}} ;
$$

that is, the duration of contact increases with the mass and decreases with the velocity of the projectile. However, for any given pad, one can find a velocity so large or a mass so small that the quasi-static static theory does not apply, as reflected in figures 7 and 8 . For a given ball size, as the velocity increases, the duration of the initial pulse decreases relative to the duration of the vibrational pulses; and for a given velocity, as the ball size decreases, the same tendency occurs. When the quasi-static theory appears to be invalid, the correlation with the predicted likelihood of injury (math model) will probably be smaller.

\subsection{Correlation Studies Using Missile Launcher}

The maximum force registered by the force transducer on the oscilloscope trace is plotted as a function of the predicted maximum nondimensional force of impact for each of the missile configurations. These plots are shown in figures 9-12 as scattergrams for each of the test pads. Using a least squares fit, regression lines have also been determined, and are shown along with the corresponding correlation coefficients on the same figures.

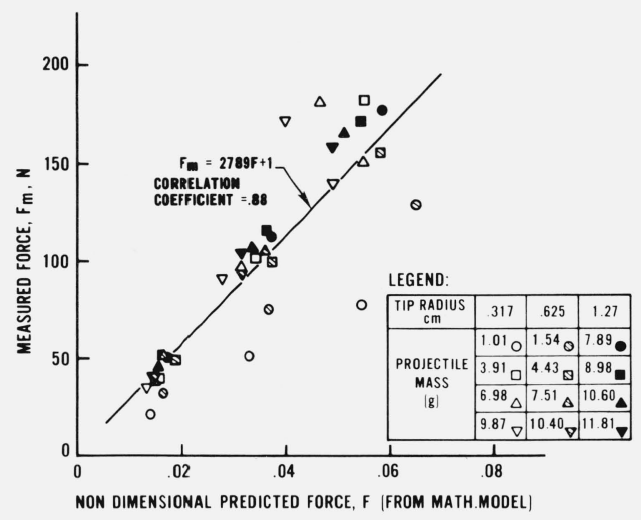

FigURE 9. Force scattergram for natural rubber pad, thickness $3.81 \mathrm{~cm}$. Each missile was fired at several different velocities.

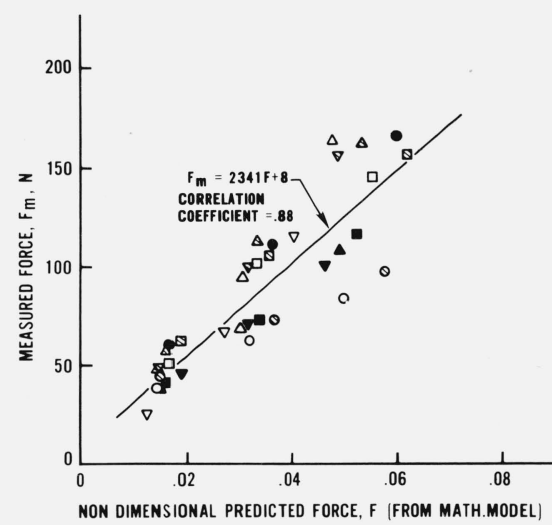

Figure 10. Force scattergram for natural rubber pad, thickness $=1.91 \mathrm{~cm}$ (legend, figure 9).

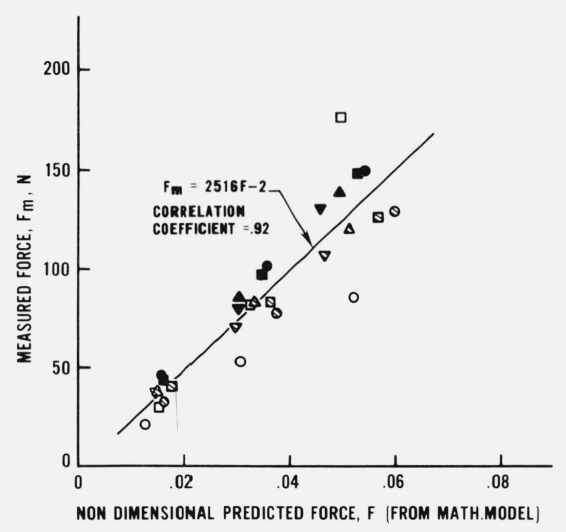

FiguRE 11. Force scattergram for natural rubber pad, thickness $=1.02 \mathrm{~cm}$ (legend, figure 9). 


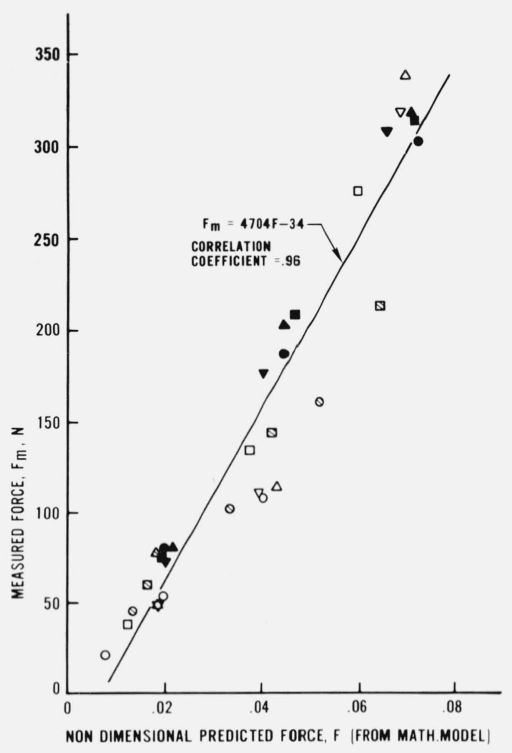

Figure 12. Force scattergram for rubber pad with carbon black compound, thickness $1.02 \mathrm{~cm}$ (legend, figure 9).

As expected, the correlation, for the natural rubber pads, improves as the pad thickness decreases. The correlation is higher still for the rubber pad with the carbon black compound. The data points for the lightweight missiles tend to fall below the other data points: this tendency increases for the thicker and softer pads and is thought to be related to the breakdown of the quasi-static theory, as discussed in the previous section.

As discussed earlier, the rate of load is related to the likelihood of eye impact injury, hence the measured and predicted times to maximum load are plotted as scattergrams for each of the pads in figures 13 to 16 . The regression and correlation coefficients are also shown on the figures. Again

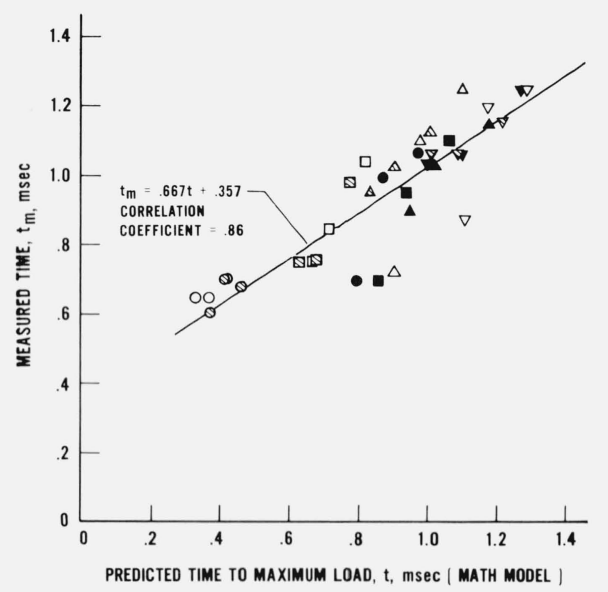

FIGURE 13. Time scattergram, natural rubber pad thickness $=3.81 \mathrm{~cm}$ (legend, figure 9).

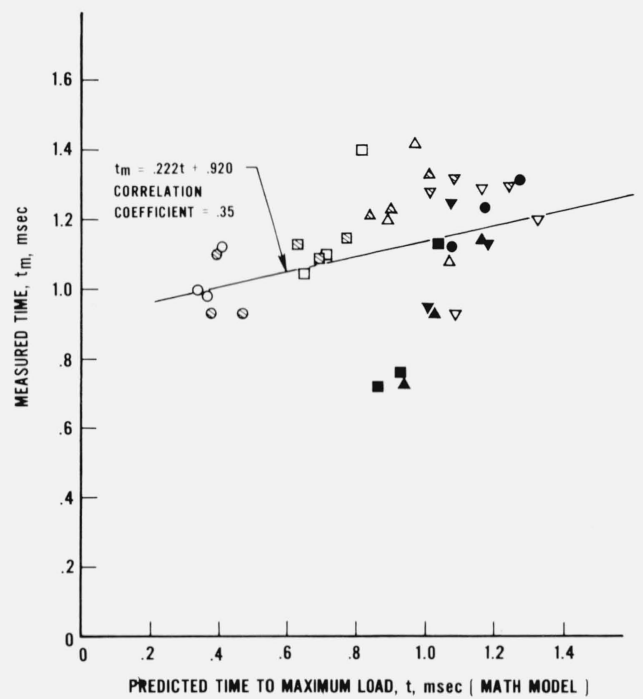

Figure 14. Time scattergram, natural rubber pad thickness $=1.91 \mathrm{~cm}$ (legend, figure 9).

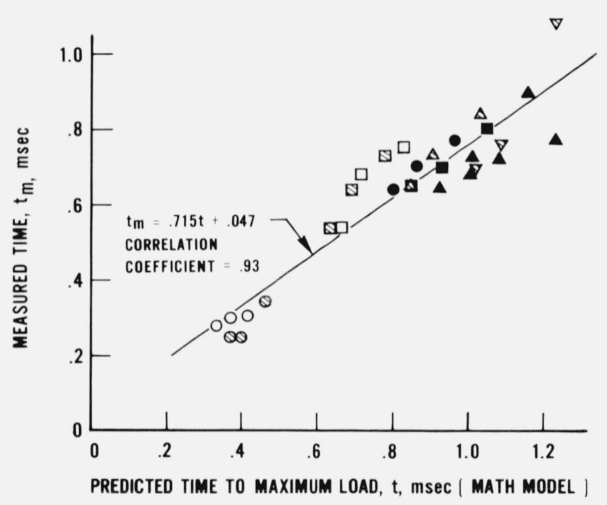

Figure 15. Time scattergram, natural rubber pad thickness $=1.02 \mathrm{~cm}$ (legend, figure 9).

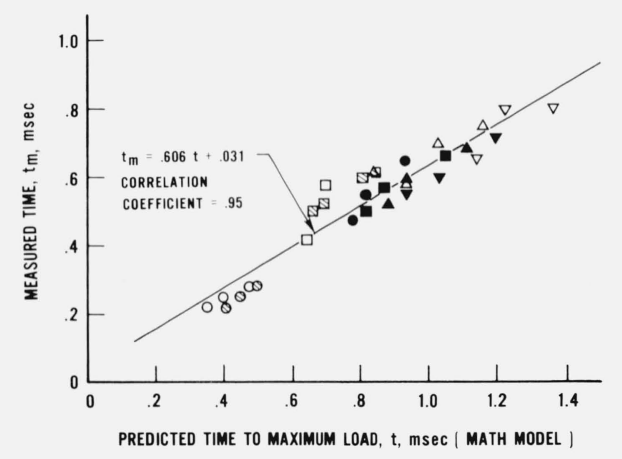

FIGURE 16. Time scattergram, rubber pad with carbon black compound thickness $=1.02 \mathrm{~cm}$ (legend, figure 9). 
the correlations are highest for the black rubber pad. The extremely poor correlation for the $1.91 \mathrm{~cm}$ thick natural rubber pad is probably due to the interaction between vibrational and static-like energy modes, as discussed in the previous section.

Since the correlation between each of the predicted injury parameters (force and time) and the corresponding measured values was highest for the pad with the carbon black rubber compound, further analysis was conducted with this set of data.

From figure 2 , it is seen that each of the tolerance curves has a slope very nearly equal to 0.8 . Since figure 2 is a $\log$ log plot, each of the injury tolerance curves can therefore be described by the equation,

$$
F=A t^{0.8}
$$

The values for $A$, for each of the injury levels of figure 2 , are presented in table 1 .

TABLE 1. Values of Injury Parameters, $A, A_{m}$ and $B$.

\begin{tabular}{l|c|c|c}
\hline Injury Level & $\begin{array}{c}A \\
\left(\mathrm{msec}^{-0.8}\right)\end{array}$ & $\begin{array}{c}A_{m} \\
\left(\mathrm{~N} / \mathrm{msec}^{0.8}\right)\end{array}$ & $\begin{array}{c}B \\
(\mathrm{~N} / \mathrm{msec})\end{array}$ \\
\hline Perforation & 0.83 & 4809 & 5889 \\
\hline Peripheral & 0.32 & 1844 & 2246 \\
\hline Chamber angle & 0.11 & 622 & 747 \\
\hline S.F. 2 & 0.055 & 303 & 354 \\
\hline
\end{tabular}

In other words, for a propelled object for which we compute $F$ and $t$, the ratio

$$
A=\frac{F}{t^{0.8}}
$$

is an index of the expected severity of injury if that object were to strike an eye. Thus, the parameter $A$ associated with a propelled object approximately weights two parameters (force and time) to form a single injury parameter. For the proposed test system, the maximum force, $F_{m}$, and the time to maximum force, $t_{m}$, are measured. The parameter,

$$
A_{m}=\frac{F_{m}}{t_{m} 0.8},
$$

has been computed and plotted as a function of $A$ (from the math model) on the scattergram of figure 17. The correlation is slightly less than the values obtained for either force or time alone.

An alternate parameter $B$, may be defined by

$$
B=\frac{F_{m}}{t_{m}} .
$$

$B$ was computed for the measured data and plotted as a function of $A$ in figure 18. The correlation here is about the same (or slightly higher) as that for $A_{m}$ with $A$. In view of the desired practicality for a test method, $B$ is recommended as simpler to compute: the decimal exponentiation can be avoided.

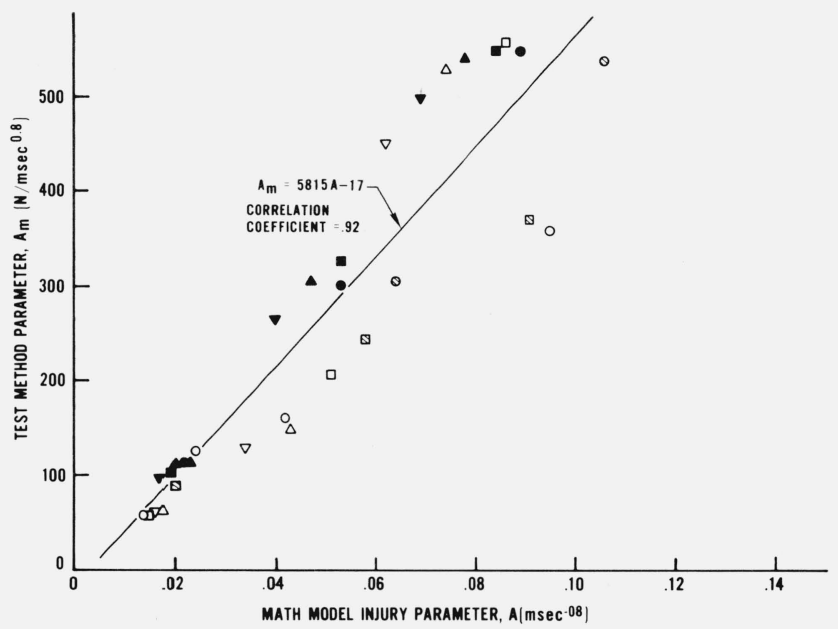

FigURE 17. $A_{m}$ vs. A, rubber pad with carbon black compound (legend, figure 9).

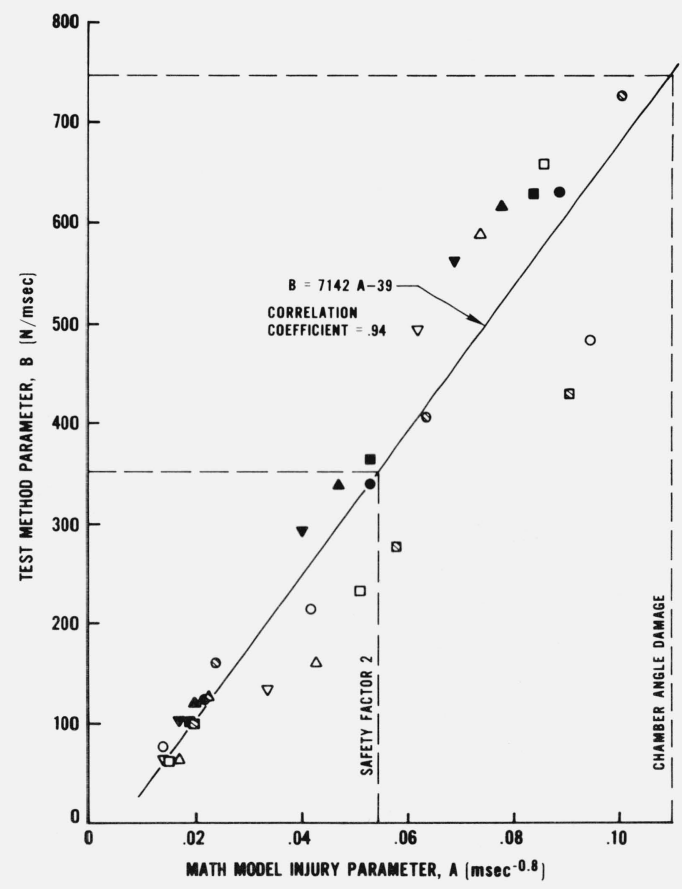

Figure 18. B vs. A, rubber pad with carbon black compound (legend, figure 9). 


\subsection{Assessing the Potential for Eye Injury}

The regression lines of figures 17 and 18 can be used to determine values of $A_{m}$ and $B$ for particular levels of injury. These are also shown in table 1. For example, the onset level for clinically detectable contusion injury (i.e., chamber angle damage) is approximately $B=750 \mathrm{~N} / \mathrm{msec}$. An unknown projectile is evaluated by impacting it on the test system, measuring the maximum force $F_{m}$ and the time to the maximum $t_{m}$ then calculating the parameter $B=F_{m} / t_{m}$. If $B$ $>750 \mathrm{~N} / \mathrm{msec}$, one could conclude that, within the limitations of the mathematical model described in the previous work [5] and the limited data on which the tolerance curves are based, the projectile poses a threat of ocular contusion.

As suggested in the earlier report, safety factors are often introduced, especially when the real life injury data base is small. The tolerance line for safety factor 2 was shown in figure 2 and the test method level of $B$ associated with it can be inferred from figure 18; that is, approximately, $B=350$ $\mathrm{N} / \mathrm{msec}$ for an injury safety factor 2 .

When the test system is to be used to assess the injury potential of propelled objects associated with toys, it is suggested that the projectile be fired from a rest position where it is already in close proximity to the pad, but subject to a requirement that the firing mechanism be allowed to operate fully. For example, the spring of a spring loaded gun should be allowed to uncoil completely. This testing situation might then be said to simulate a worst case, say where a child holds the projectile toy near his eye and accidentally discharges it. On the other hand, the accuracy with which the projectile strikes the test system is not a critical concern. The response of the test system to impacts which strike halfway to the edge of the pad is nearly the same as for those striking the center. A toy which is about to be fired into the test system is shown in figure 19.

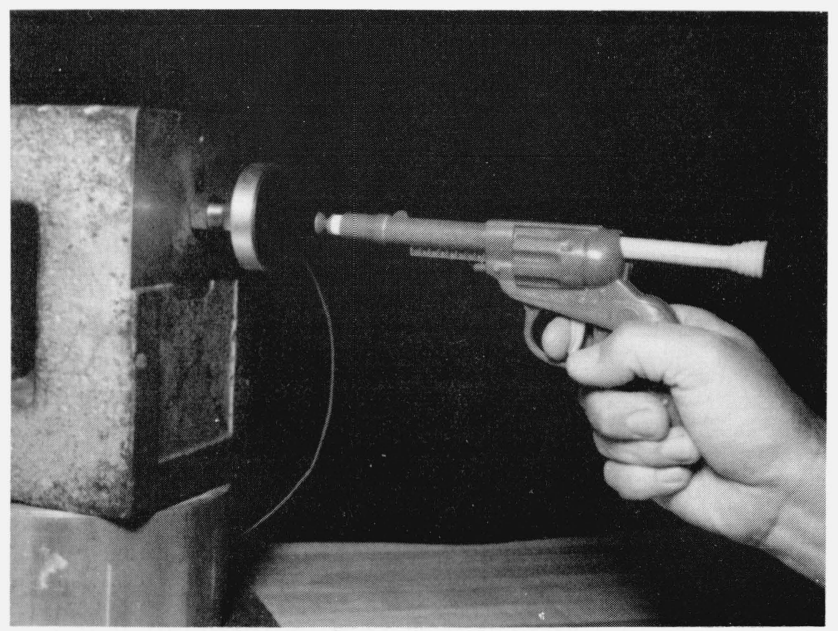

Figure 19. Photo of toy about to be fired into test system.
A group of toy projectiles, described in table 2, was tested in the above manner; a few typical oscilloscope traces are shown in figure 20. Review of the oscilloscope traces reveals several classes of response. Many projectiles which are nearly rigid and which have regular geometries respond in a manner similar to that of the test missiles and steel balls: the

Table 2. Projectile Toys Tested in Accordance with Proposed Method.

\begin{tabular}{|c|c|c|c|c|}
\hline $\begin{array}{l}\text { Toy } \\
\text { ID }\end{array}$ & Description & $F_{m}(\mathrm{~N})$ & $t_{m}(\mathrm{msec})$ & $B(\mathrm{~N} / \mathrm{msec})$ \\
\hline 13 & Ping pong ball shooter & 107 & 0.25 & 428 \\
\hline 22 & Machine gun, plastic bullet & 93 & .27 & 344 \\
\hline 48 & Machine gun, plastic bullet & 19 & .20 & 95 \\
\hline 20 & $\begin{array}{l}\text { Gun, bullet has suction cup at- } \\
\text { tached }\end{array}$ & 91 & .15 & 607 \\
\hline 33 & Rifle, small rubbery bullet & 24 & .25 & 96 \\
\hline 26 & Cork gun & 64 & .20 & 320 \\
\hline 15 & Cork gun & 53 & .10 & 530 \\
\hline 47 & Dart gun with suction cups & 224 & .25 & 896 \\
\hline 7 & Dart gun with suction cups & 107 & .40 & 267 \\
\hline 49 & Dart gun with suction cups & 176 & .25 & 704 \\
\hline 25 & Dart gun with suction cups & 48 & .45 & 107 \\
\hline 41 & Plastic disc shooter & 24 & .20 & 120 \\
\hline 46 & Plastic disc shooter & 32 & .15 & 213 \\
\hline \multirow[t]{3}{*}{44} & Aircraft carrier & & & \\
\hline & A Flexible planes with rub- & 192 & .30 & 640 \\
\hline & B mechanism & 133 & 1.00 & 133 \\
\hline \multirow[t]{5}{*}{50} & Warrior, shoots various objects: & & & \\
\hline & A fist & 267 & 1.35 & 198 \\
\hline & B ax & 48 & 0.6 & 80 \\
\hline & C bullet & 43 & .6 & 72 \\
\hline & D airplane & 59 & .6 & 98 \\
\hline
\end{tabular}

(a) 13

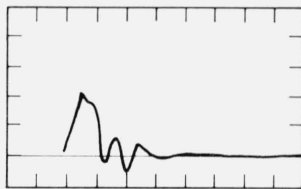

(b) $50 \mathrm{~A}$

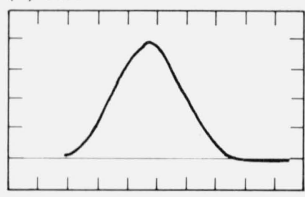

(c) 33

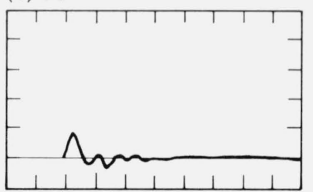

(d) 49

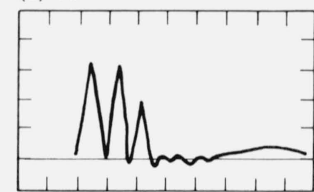

(e) 47

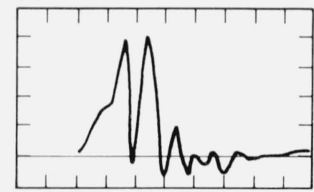

(f) $44 \mathrm{~A}$

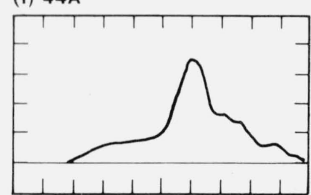

FigurE 20. Sketches of oscilloscope traces following the impact of selected toy projectiles with the test system.

Vertical scale $53.4 \mathrm{~N}$ per division; horizontal scale $0.5 \mathrm{msec}$ per div. 
force increases for a time and then decreases, as in figures 20a, b and c. Others have more peculiar responses: the double peaked traces of figures $20 \mathrm{~d}$ and e are typical of the responses from darts with suction cups. It is conjectured that the first peak is related to the impact of the flexible tip, and that the second peak is related to the impact of the stem after the flexible part has bottomed. (In fact, the second peak may be larger than the first.) Figure 20f is an example of a completely flexible missile, which tends to bend on impact. The force stays at a very low level for some time, then suddenly increases to its maximum before dropping off. The sudden rise in force presumably coincides with the completion of the missile's bending phase. The energy expended in bending is no longer available for deforming the pad, hence the resulting maximum force is less than it would have been if the missile were rigid.

The examples of figures 20e and $\mathrm{f}$ highlight an undesirable limitation in the computation of $B$ : the length of time to the maximum force $\left(t_{m}\right.$, which appears in the denominator of $B$ ) may misrepresent the load rate if measured from the onset of impact. Rather, $F_{m}$ and $t_{m}$ should be measured within each pulse which exhibits a relative maximum force; $B$ should then be computed for each pulse and the largest value selected to represent the injury likelihood index. The beginning of a pulse is determined by inspection or by extrapolation, if necessary, as shown in figure 21 .

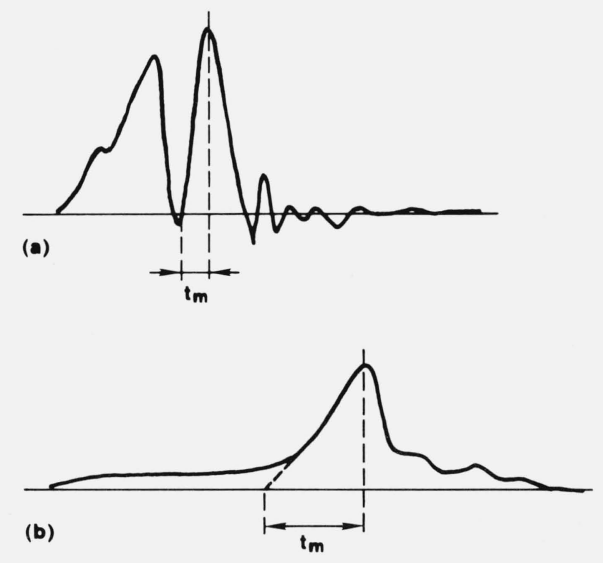

Figure 21. Method of determining $t_{m}$ by extrapolation.

For each toy projectile tested, the parameters $F_{m}$ and $t_{m}$ were measured from the oscilloscope traces; these values, along with $B$ are shown in table 2 . Alternatively, figures 12 and 16 can be used to determine $F$ and $t$ when $F_{m}$ and $t_{m}$ are measured. A plot of corresponding data points can be superimposed on the injury tolerance curves (of fig. 2), as shown in figure 22 , for the projectiles listed in table 2 .

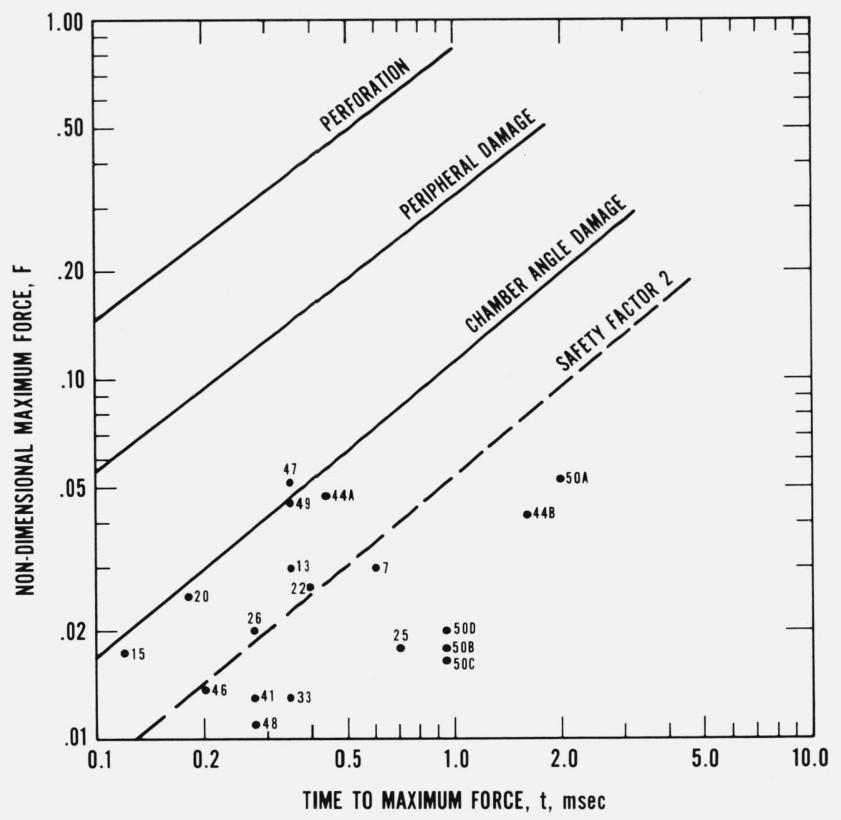

Figure 22. Relation of selected toy projectiles to tolerance curves.

\subsection{Recommendations and Limitations}

The test system utilizing the rubber pad with the carbon black compound (ASTM D15-73, compound 7A) is recommended for use in evaluating the injury potential of low speed propelled objects. It has been shown that a satisfactory correlation exists between the likelihood of ocular contusion injury, as predicted by a mathematical model, and the response of this impact test system. The response was related to ocular injury tolerance curves which were generated with a mathematical model developed from data on impact injuries to real eyes. The tolerance curves were further found to agree with the subjective opinions of ophthalmologists.

It must be emphasized that this test system is related to the likelihood of contusion injuries. In particular, test results do not apply to corneal abrasion injuries which may be associated with propelled objects with non-blunt potential impact surfaces. Any propelled object with a well defined edge is probably sharp enough to abrade the cornea at even very small forces of impact. However, such injuries are usually considered to be reversible. For example, such abrasions are not uncommon among contact lens wearers.

Other limitations should also be noted: (1) the injury data on which the tolerance curves are based is very limited; (2) an experimental verification of the applicability of the quasistatic theory would strengthen the case for the suitability of the simulation. To address both of these concerns, high speed motion picture experiments with real enucleated eyes are in progress, and are expected to be the subject of a future paper. 
I thank Harvey Kratz for his thoughtful design and construction of the apparatus and also Jim Huckeba, Laura Nesbitt, Ray Russell, Louise Shannon, and Marriane Vaishnav for their assistance.

\section{References}

[1] Jensen, O. A., Contusive Angle Recession, A Histolopathological Study of a Danish Material, Acta Ophthalmologica, 46, 1207-1212 (1968).

[2] Wolff, S. M. and Zimmerman, L. E., Chronic Secondary Glaucoma,
Associated with Retrodisplacement of Iris Root and Deepening of the Anterior Chamber Angle Secondary to Contusion, Am. J. Ophthal., 54, 547-562 (1962).

[3] Weidenthal, D. T. and Schepens, C. L., Peripheral Fundus Changes Associated with Ocular Contusion, Am. J. Ophthal. 62, 465-477 (1966).

[4] Tonjum, A. M., Lens Dislocation Following Ocular Contusion, Acta Ophthalmologica 46, 860-873 (1968).

[5] Berger, R. E., A Model for Evaluating the Ocular Contusion Injury Potential of Propelled Objects, Journal of Bioengineering, 2, 3 345-358 (1978).

[6] Fischler, S. and Mahajan, B. M., Ocular Injury Potential of ProjectileType Toys, National Bureau of Standards Technical Report, 10893 (1972).

[7] Goldsmith, W., Impact, p. 90, Edward Arnold Publishers, London (1960). 